\title{
Organocatalysis for the synthesis of optically active $\beta$-malonophosphonates
}

\author{
PRASHANT B THORAT, SANTOSH V GOSWAMI, RUPALI L MAGAR \\ and SUDHAKAR R BHUSARE* \\ Department of Chemistry, Dnyanopasak College, Parbhani 431401, India \\ e-mail: bhusare71@yahoo.com
}

MS received 19 February 2013; revised 12 June 2013; accepted 4 July 2013

\begin{abstract}
Method describes asymmetric reaction of triethyl phosphites and 2-arylidenemalononitrile catalysed by the chiral organocatalyst in solvent ethanol. The organocatalyst effectively catalyse reaction to give $\beta$-malonophosphonates in high yields (85-64\%) and good to moderate enantiomeric excess (78-53\%).
\end{abstract}

Keywords. Asymmetric synthesis; $\beta$-malonophosphonates; chiral organocatalyst; enantioselectivity; hydrogen bonding

\section{Introduction}

The asymmetric synthesis of organophosphorus compounds is not received much attention in the organic synthetic chemistry. Organophosphorus molecules constitute an important class of pharmacologically active molecules. ${ }^{1}$ These molecules are useful for the treatment of bone disorders, such as myeloma, Paget's disease, bone metastases and rheumatoid arthritis. ${ }^{2}$ Some of the chiral organophosphporus are also used as ligands in asymmetric synthesis. ${ }^{3}$ In addition, enantiopure natural products containing a $\mathrm{P}-\mathrm{C}$ bond mostly exhibit important biological activities. ${ }^{4}$

Direct phosphorus-carbon bond formation represents one of the most versatile and powerful tools for the synthesis of phosphonates. Phospha-Michael addition is the addition of a phosphorous nucleophile to an electron-deficient alkene and received remarkable attention from organic chemists. ${ }^{5}$ Arbuzov in the early 20 th century worked on various reaction for the formation of $\mathrm{P}-\mathrm{C}$ bond, which are well-known as MichaeliesArbuzov reactions. ${ }^{6}$ With the discovery of naturally occurring aminophosphonic acid and phosphonate this field gained more importance. ${ }^{5}$

Amongst various methods to generate phosphoruscarbon bond, addition of $\mathrm{P}(\mathrm{O}) \mathrm{H}$ bond across alkene is the most famous. Further these reactions are most commonly promoted by bases, ${ }^{7}$ acids,${ }^{8}$ microwaves, ${ }^{9}$ alkaline metal oxides, ${ }^{2,10}$ tetramethylguanidine ${ }^{11}$ or radical initiators such as AIBN. ${ }^{12}$ It is also achieved by nanosized $\mathrm{ZnO},{ }^{13}$ heterogeneous solid, ${ }^{14}$ Phosphomolybdic

*For correspondence acid, hydrophosphorylation of unactivated alkene catalysed by transition metals. ${ }^{15,16}$ Michael additions to activated vinylphosphonates is another method to obtain $\beta$-phosphono malonates. ${ }^{17}$ Recently Kolla et al. reported organocatalytic synthesis of $\beta$-phosphono malonates by using ethylenediamine diacetate (EDDA) as a catalyst. ${ }^{18}$ Above literature survey towards the synthesis of $\beta$-phosphono malonates revealed that discussion on asymmetric synthesis is rare.

Although these methods are valuable, they suffer from one or more of the following disadvantages hazardous conditions, long reaction times, low yields, tedious work-up protocols, requiring a promoter such as microwave and using large amounts of un-recyclable catalyst which would eventually result in the generation of a large amount of toxic waste. Hence, the development of a new and efficient catalyst for this important transformation is still in demand.

Thus, owing to the synthetic and biological values of phosphonates, synthesis of phosphorus containing molecules is one of the important fields that continuing to attract the attention of organic chemists. We describe here the use of chiral organocatalyst for the synthesis of $\beta$-malonophosphonates. We also studied enantioslectivity of obtained products.

\section{Experimental}

\subsection{General details}

All solvents were used as commercial grade without further purification. Aluminium sheets $20 \times 20 \mathrm{~cm}$, 
silica gel $60 \mathrm{~F}_{254}$, Merck grade was used for thin-layer chromatography to determine progress of reaction. The column chromatography (TLC) was carried out over silica gel (80-120 mesh). Optical rotations were measured on a Polax-2L digital polarimeter at $27^{\circ} \mathrm{C}$. Melting points were determined in open capillary tube and are uncorrected. ${ }^{1} \mathrm{H}$ and ${ }^{13} \mathrm{C}$ NMR spectra were recorded on a Bruker $300 \mathrm{MHz}$ spectrometer in $\mathrm{CDCl}_{3}$ solvent. Mass spectra were taken on Polaris-Q Thermoscintific GC-MS. Enantiomeric purity is determined on PerkinElmer Series 200 HPLC Systems using chiral Whelk-O1 $(25 \mathrm{~cm} \times 4.6 \mathrm{~mm}), 10 \% \mathrm{EtOH} /$ hexane, Flow rate $1.0 \mathrm{~mL} / \mathrm{min}, \lambda=245 \mathrm{~nm}$; samples peaks are confirmed from commercially available racemic mixture.

\subsection{General procedure for the synthesis} of organocatalyst 1 and 2

The synthesis and characterization of the organocatalysts $\mathbf{1}$ and $\mathbf{2}$ is reported in our earlier communications. ${ }^{19}$

\subsection{General procedure for synthesis of $\beta$-phosphonomalonates}

A mixture of alkylidinemalanonitrile $(1 \mathrm{mmol})$, trimethyl phosphite $(1.2 \mathrm{mmol})$ and organocatalyst $(S)$ 1-acetylpyrrolidine-2-carboxamide $(12 \mathrm{~mol} \%)$ were added to reaction flask containing ethanol as solvent. The reaction mixture was refluxed for the appropriate time. The progress of the reaction was monitored by TLC. After completion of the reaction as indicated by TLC, the mixture was allowed to attain room temperature. To this cooled reaction mixture, ice cold water was added and extracted with ethyl acetate. The organic layer was collected, washed with brine solution and dried over anhydrous sodium sulphate. The solvent was removed under vacuum to obtain crude product, which was purified by column chromatography to afford the pure crystals of $\beta$-phosphonomalonates. The desired products obtained were characterized by ${ }^{1} \mathrm{H}$ NMR, ${ }^{13} \mathrm{C}$ NMR, ${ }^{31} \mathrm{P}$ NMR and GC-MS spectra and chiral HPLC technique (please refer to supporting information for details).

\subsection{Characterization}

2.4a Diethyl 1-(4-chlorophenyl)-2, 2-dicyanoethylphosphonate (5a): White solid; (Yield $295 \mathrm{mg}$; 85\%), M. P. $159-160^{\circ} \mathrm{C},[\alpha]_{\mathrm{D}}-25.6\left(\mathrm{c} 1.0, \mathrm{CHCl}_{3}\right) ;{ }^{1} \mathrm{H} \mathrm{NMR}$ (300 MHz, $\left.\mathrm{CDCl}_{3}\right): \delta 7.40-7.61(\mathrm{~m}, 2 \mathrm{H}), 7.21-7.37$ (m, $2 \mathrm{H}), 4.34\left(\mathrm{t}, J^{\mathrm{H}-\mathrm{H}, \mathrm{H}-\mathrm{P}}=10.1 \mathrm{~Hz}, 7.9 \mathrm{~Hz}, 1 \mathrm{H}\right), 3.98(\mathrm{~m}$, $4 \mathrm{H}), 3.53\left(\mathrm{dd}, J^{\mathrm{H}-\mathrm{P}}=22.1 \mathrm{~Hz}, J^{\mathrm{H}-\mathrm{H}}=7.4 \mathrm{~Hz}, 1 \mathrm{H}\right)$, $1.14\left(\mathrm{t}, J^{\mathrm{H}-\mathrm{H}}=7.6 \mathrm{~Hz}, 3 \mathrm{H}\right), 0.93\left(\mathrm{t}, J^{\mathrm{H}-\mathrm{H}}=7.6 \mathrm{~Hz}\right.$,
3H); ${ }^{13} \mathrm{C}-\mathrm{NMR}\left(75 \mathrm{MHz}, \mathrm{CDCl}_{3}\right): \delta$ 136.06, 135.49, $132.46,130.01,114.94\left(\mathrm{~d}, J^{\mathrm{C}-\mathrm{P}}=10.6 \mathrm{~Hz}\right), 114.53(\mathrm{~d}$, $\left.J^{\mathrm{C}-\mathrm{P}}=9.3 \mathrm{~Hz}\right), 62.89\left(\mathrm{~d}, J^{\mathrm{C}-\mathrm{P}}=7.3 \mathrm{~Hz}\right), 62.31(\mathrm{~d}$, $\left.J^{\mathrm{C}-\mathrm{P}}=7.3 \mathrm{~Hz}\right), 44.43\left(\mathrm{~d}, J^{\mathrm{C}-\mathrm{P}}=144.8 \mathrm{~Hz}\right), 29.00$, $16.51\left(\mathrm{~d}, J^{\mathrm{C}-\mathrm{P}}=5.1 \mathrm{~Hz}\right), 16.10\left(\mathrm{~d}, J^{\mathrm{C}-\mathrm{P}}=5.8 \mathrm{~Hz}\right)$; ${ }^{31} \mathrm{P}$ NMR $\left(162 \mathrm{MHz}, \mathrm{CDCl}_{3}\right.$ ): $\delta$ 20.12; GC-MS: $\mathrm{m} / \mathrm{z}$ $326(\mathrm{M}+)$; Elemental Analysis: $\mathrm{C}_{14} \mathrm{H}_{16} \mathrm{ClN}_{2} \mathrm{O}_{3} \mathrm{P}: \mathrm{C}$, 51.47; H, 4.94; Cl, 10.85; N, 8.57; P, 9.48; Found C, 51.45; H, 4.93; Cl, 10.83; N, 8.55; 14.71; P, 9.47. HPLC: $78 \%$ ee. [Determined by chiral HPLC with Whelk-O1 $(25 \mathrm{~cm} \times 4.6 \mathrm{~mm}), 10 \% \mathrm{EtOH} / \mathrm{Hexane}$, Flow rate $1.0 \mathrm{~mL} / \mathrm{min}, \lambda=245 \mathrm{~nm} ; \mathrm{t}_{\mathrm{R}}$ (major) $=$ $35.0 \mathrm{~min}, \mathrm{t}_{\mathrm{R}}($ minor $\left.)=36.3 \mathrm{~min}\right]$.

2.4b Diethyl 2, 2-dicyano-1-(4-nitrophenyl)ethylphosphonate $(5 \boldsymbol{b})$ : Yellow solid, M. P. $121-123^{\circ} \mathrm{C},[\alpha]_{\mathrm{D}}$ -13.7 (c 1.0, $\mathrm{CHCl}_{3}$ ); the title compound was prepared according to the general procedure, as described above in $(281 \mathrm{mg}), 83 \%$ yield. The structure of compound was confirmed by comparing spectral data, which were found to be identical with those described in literature 13a. HPLC: $71 \%$ ee. [Determined by chiral HPLC with Whelk-O1 $(25 \mathrm{~cm} \times 4.6 \mathrm{~mm}), 10 \% \mathrm{EtOH} /$ Hexane, Flow rate $1.0 \mathrm{~mL} / \mathrm{min}, \lambda=245 \mathrm{~nm} ; \mathrm{t}_{\mathrm{R}}$ (major) $=$ $29.6 \mathrm{~min}, \mathrm{t}_{\mathrm{R}}($ minor $\left.)=33.8 \mathrm{~min}\right]$.

\section{4c Diethyl 2, 2-dicyano-1-(3-nitrophenyl)ethylphos-} phonate (5c): Yellowish solid, (Yield $288 \mathrm{mg}$; 85\%), M. P. $136-138^{\circ} \mathrm{C},[\alpha]_{\mathrm{D}}-21.8\left(\mathrm{c} 1.0, \mathrm{CHCl}_{3}\right) ;{ }^{1} \mathrm{H} \mathrm{NMR}$ (300 MHz, $\left.\mathrm{CDCl}_{3}\right)$ : 7.38-7.56 (m, 2H), 6.63-6.98 (m, $2 \mathrm{H}), 4.49\left(\mathrm{t}, J^{\mathrm{H}-\mathrm{H}, \mathrm{H}-\mathrm{P}}=9.8 \mathrm{~Hz}, 1 \mathrm{H}\right), 4.01(\mathrm{~m}, 4 \mathrm{H})$, $3.59\left(\mathrm{dd}, J^{\mathrm{H}-\mathrm{P}}=21.8 \mathrm{~Hz}, J^{\mathrm{H}-\mathrm{H}}=7.7 \mathrm{~Hz}, 1 \mathrm{H}\right), 1.13$ $\left(\mathrm{t}, J^{\mathrm{H}-\mathrm{H}}=6.8 \mathrm{~Hz}, 3 \mathrm{H}\right), 0.89\left(\mathrm{t}, J^{\mathrm{H}-\mathrm{H}}=6.8 \mathrm{~Hz}\right.$, $3 \mathrm{H}) ;{ }^{13} \mathrm{C}-\mathrm{NMR}\left(75 \mathrm{MHz}, \mathrm{CDCl}_{3}\right): \delta 150.09,143.02$, 133.08, 122.98, 114.26, 113.88, 65.79, 65.14, 43.14, 28.88, 16.51, 16.10; ${ }^{31} \mathrm{P}$ NMR $\left(162 \mathrm{MHz}, \mathrm{CDCl}_{3}\right): \delta$ 22.31; GC-MS: $m / z 337(\mathrm{M}+)$; Elemental Analysis: $\mathrm{C}_{14} \mathrm{H}_{16} \mathrm{~N}_{3} \mathrm{O}_{5} \mathrm{P}: \mathrm{C}, 49.86 ; \mathrm{H}, 4.78 ; \mathrm{N}, 12.46$; P, 9.18; Found C, 49.84; H, 4.81; N, 12.43; P, 9.21. HPLC: $67 \%$ ee. [Determined by chiral HPLC with Whelk-O1 $(25 \mathrm{~cm} \times 4.6 \mathrm{~mm}), 10 \% \mathrm{EtOH} / \mathrm{Hexane}$, Flow rate $1.0 \mathrm{~mL} / \mathrm{min}, \lambda=245 \mathrm{~nm} ; \mathrm{t}_{\mathrm{R}}$ (major) $=16.7 \mathrm{~min}, \mathrm{t}_{\mathrm{R}}$ $(\operatorname{minor})=41.9 \mathrm{~min}]$.

2.4d Diethyl 2, 2-dicyano-1-(4-fluorophenyl)ethylphosphonate (5d): White solid, (Yield $292 \mathrm{mg} ; 81 \%$ ), M. P. $169-171^{\circ} \mathrm{C},[\alpha]_{\mathrm{D}}-15.9\left(\mathrm{c} 1.0, \mathrm{CHCl}_{3}\right) ;{ }^{1} \mathrm{H}$ NMR (300 MHz, $\left.\mathrm{CDCl}_{3}\right): 7.19-7.32(\mathrm{~m}, 2 \mathrm{H}), 6.79-6.90(\mathrm{~m}$, $2 \mathrm{H}), 4.52\left(\mathrm{t}, J^{\mathrm{H}-\mathrm{H}, \mathrm{H}-\mathrm{P}}=10.3 \mathrm{~Hz}, 1 \mathrm{H}\right), 4.11(\mathrm{~m}, 4 \mathrm{H})$, $3.58\left(\mathrm{dd}, J^{\mathrm{H}-\mathrm{P}}=19.8 \mathrm{~Hz}, J^{\mathrm{H}-\mathrm{H}}=6.5 \mathrm{~Hz}, 1 \mathrm{H}\right), 1.21$ $\left(\mathrm{t}, J^{\mathrm{H}-\mathrm{H}}=6.8 \mathrm{~Hz}, 3 \mathrm{H}\right), 1.07\left(\mathrm{t}, J^{\mathrm{H}-\mathrm{H}}=6.8 \mathrm{~Hz}, 3 \mathrm{H}\right)$; ${ }^{13} \mathrm{C}-\mathrm{NMR}\left(75 \mathrm{MHz}, \mathrm{CDCl}_{3}\right): \delta 160.00,128.15,127.90$, $115.89,113.89\left(\mathrm{~d}, J^{\mathrm{C}-\mathrm{P}}=12.7 \mathrm{~Hz}\right), 113.27\left(\mathrm{~d}, J^{\mathrm{C}-\mathrm{P}}=\right.$ 
$11.5 \mathrm{~Hz}), 64.90\left(\mathrm{~d}, J^{\mathrm{C}-\mathrm{P}}=7.3 \mathrm{~Hz}\right), 64.37\left(\mathrm{~d}, J^{\mathrm{C}-\mathrm{P}}=\right.$ $6.9 \mathrm{~Hz}), 41.93\left(\mathrm{~d}, J^{\mathrm{C}-\mathrm{P}}=142.7 \mathrm{~Hz}\right), 27.00,16.27(\mathrm{~d}$, $\left.J^{\mathrm{C}-\mathrm{P}}=5.1 \mathrm{~Hz}\right), 15.94\left(\mathrm{~d}, J^{\mathrm{C}-\mathrm{P}}=4.9 \mathrm{~Hz}\right) ;{ }^{31} \mathrm{P} \mathrm{NMR}$ $\left(162 \mathrm{MHz}, \mathrm{CDCl}_{3}\right)$ : $\delta$ 22.44; GC-MS: $m / z 310(\mathrm{M}+)$; Elemental Analysis: $\mathrm{C}_{14} \mathrm{H}_{16} \mathrm{FN}_{2} \mathrm{O}_{3} \mathrm{P}: \mathrm{C}, 54.20 ; \mathrm{H}, 5.20$; F, 6.12; N, 9.03; P, 9.98; Found C, 54.23; H, 5.21; F, $6.09 ; \mathrm{N}, 9.01 ; \mathrm{P}, 10.01$. HPLC: 69\% ee. [Determined by chiral HPLC with Whelk-O1 $(25 \mathrm{~cm} \times 4.6 \mathrm{~mm}), 10 \%$ $\mathrm{EtOH} /$ Hexane, Flow rate $1.0 \mathrm{~mL} / \mathrm{min}, \lambda=245 \mathrm{~nm} ; \mathrm{t}_{\mathrm{R}}$ $($ minor $)=32.0 \mathrm{~min}, \mathrm{t}_{\mathrm{R}}($ major $\left.)=36.5 \mathrm{~min}\right]$.

2.4e Diethyl 1-(3-chlorophenyl)-2, 2-dicyanoethylphosphonate (5e): White solid, M. P. $148-150^{\circ} \mathrm{C}$, $[\alpha]_{\mathrm{D}}-36.5\left(\mathrm{c} 1.0, \mathrm{CHCl}_{3}\right)$; the title compound was prepared according to the general procedure, as described above in $(271 \mathrm{mg}) 78 \%$ yield. The structure of compound was confirmed by comparing spectral data, which were found to be identical with those described in literature 13a. HPLC: $76 \%$ ee. [Determined by chiral HPLC with Whelk-O1 $(25 \mathrm{~cm} \times 4.6 \mathrm{~mm}), 10 \%$ $\mathrm{EtOH} / \mathrm{Hexane}$, Flow rate $1.0 \mathrm{~mL} / \mathrm{min}, \lambda=245 \mathrm{~nm}$; $\mathrm{t}_{\mathrm{R}}$ $($ major $)=24.6 \mathrm{~min}, \mathrm{t}_{\mathrm{R}}($ minor $\left.)=32.4 \mathrm{~min}\right]$.

$2.4 \mathrm{f}$ Diethyl 2, 2-dicyano-1-(4-hydroxyphenyl) ethylphosphonate (5f): Colourless solid, M. P. $176-178^{\circ} \mathrm{C}$, $[\alpha]_{\mathrm{D}}-41.6\left(\mathrm{c} 1.0, \mathrm{CHCl}_{3}\right)$; the title compound was prepared according to the general procedure, as described above in $(308 \mathrm{mg})$ 85\% yield. The structure of compound was confirmed by comparing spectral data, which were found to be identical with those described in literature 14. HPLC: $59 \%$ ee. [Determined by chiral HPLC with Whelk-O1 $(25 \mathrm{~cm} \times 4.6 \mathrm{~mm}), 10 \%$ $\mathrm{EtOH} /$ Hexane, Flow rate $1.0 \mathrm{~mL} / \mathrm{min}, \lambda=245 \mathrm{~nm}$; $\mathrm{t}_{\mathrm{R}}$ $($ major $)=18.9 \mathrm{~min}, \mathrm{t}_{\mathrm{R}}($ minor $\left.)=29.7 \mathrm{~min}\right]$.

2.4g Diethyl 2, 2-dicyano-1-(2-hydroxyphenyl) ethylphosphonate (5g): Colourless solid, (Yield $299 \mathrm{mg}$; $80 \%$ ), M. P. $167-169^{\circ} \mathrm{C},[\alpha]_{\mathrm{D}}+38.2$ (c 1.0, $\mathrm{CHCl}_{3}$ ); ${ }^{1} \mathrm{H}$ NMR $\left(300 \mathrm{MHz}, \mathrm{CDCl}_{3}\right): \delta 8.19(\mathrm{~s}, 1 \mathrm{H}, \mathrm{OH}), 7.25-$ $7.31(\mathrm{~m}, 1 \mathrm{H}), 6.88-6.96(\mathrm{~m}, 1 \mathrm{H}), 6.57-6.69(\mathrm{~m}, 2 \mathrm{H})$, $4.58\left(\mathrm{t}, J^{\mathrm{H}-\mathrm{H}, \mathrm{H}-\mathrm{P}}=9.9 \mathrm{~Hz}, 1 \mathrm{H}\right), 4.06(\mathrm{~m}, 4 \mathrm{H}), 3.62$ $\left(\mathrm{dd}, J^{\mathrm{H}-\mathrm{P}}=20.5 \mathrm{~Hz}, J^{\mathrm{H}-\mathrm{H}}=7.2 \mathrm{~Hz}, 1 \mathrm{H}\right), 1.29(\mathrm{t}$, $\left.J^{\mathrm{H}-\mathrm{H}}=6.8 \mathrm{~Hz}, 3 \mathrm{H}\right), 1.17\left(\mathrm{t}, J^{\mathrm{H}-\mathrm{H}}=6.8 \mathrm{~Hz}, 3 \mathrm{H}\right) ;{ }^{13} \mathrm{C}-$ NMR (75 MHz, $\left.\mathrm{CDCl}_{3}\right): \delta 157.3,143.1,126.5,125.5$, 124.7, 120.8, 113.7, 113.2, 63.1, 61.4, 42.0, 28.8, 16.4, $16.3 ;{ }^{31} \mathrm{P}$ NMR (162 MHz, $\left.\mathrm{CDCl}_{3}\right): \delta$ 19.36; GC-MS: $m / z 308(\mathrm{M}+)$; Elemental Analysis: $\mathrm{C}_{14} \mathrm{H}_{17} \mathrm{~N}_{2} \mathrm{O}_{4} \mathrm{P}: \mathrm{C}$, 54.55; H, 5.56; N, 9.09; , 10.05; Found C, 54.51; H, 5.59; N, 9.11; P, 10.07. HPLC: 63\% ee. [Determined by chiral HPLC with Whelk-O1 $(25 \mathrm{~cm} \times 4.6 \mathrm{~mm}), 10 \%$ $\mathrm{EtOH} / \mathrm{Hexane}$, Flow rate $1.0 \mathrm{~mL} / \mathrm{min}, \lambda=245 \mathrm{~nm}$; $\mathrm{t}_{\mathrm{R}}$ $($ minor $)=27.6 \mathrm{~min}, \mathrm{t}_{\mathrm{R}}($ major $\left.)=39.7 \mathrm{~min}\right]$. 2.4h Diethyl 2, 2-dicyano-1-phenylethylphosphonate $(5 \boldsymbol{h})$ : White solid, M. P. $119-121^{\circ} \mathrm{C},[\alpha]_{\mathrm{D}}-12.7$ (c 1.0, $\mathrm{CHCl}_{3}$ ); the title compound was prepared according to the general procedure, as described above in $(292 \mathrm{mg}$ ) $77 \%$ yield. The structure of compound was confirmed by comparing spectral data, which were found to be identical with those described in literature 13a. HPLC: $59 \%$ ee [Determined by chiral HPLC with Whelk-O1 $(25 \mathrm{~cm} \times 4.6 \mathrm{~mm}), 10 \% \mathrm{EtOH} /$ hexane, flow rate $1.0 \mathrm{~mL} / \mathrm{min}, \lambda=245 \mathrm{~nm} ; \mathrm{t}_{\mathrm{R}}$ (major) $=23.6 \mathrm{~min}$, $\left.\mathrm{t}_{\mathrm{R}}(\operatorname{minor})=30.9 \mathrm{~min}\right]$.

2.4i Diethyl 2, 2-dicyano-1-p-tolylethylphosphonate (5i): Yellow solid, M. P. $179-181^{\circ} \mathrm{C},[\alpha]_{\mathrm{D}}-64.3$ (c 1.0, $\mathrm{CHCl}_{3}$ ) the title compound was prepared according to the general procedure, as described above in $(288 \mathrm{mg})$ $79 \%$ yield. The structure of compound was confirmed by comparing spectral data, which were found to be identical with those described in literature 13a. HPLC: $65 \%$ ee. [Determined by chiral HPLC with WhelkO1 $(25 \mathrm{~cm} \times 4.6 \mathrm{~mm}), 10 \% \mathrm{EtOH} /$ hexane, flow rate $1.0 \mathrm{~mL} / \mathrm{min}, \lambda=245 \mathrm{~nm} ; \mathrm{t}_{\mathrm{R}}$ (major) $=29.3 \mathrm{~min}, \mathrm{t}_{\mathrm{R}}$ $($ minor $)=34.5 \mathrm{~min}]$.

2.4j Diethyl 2, 2-dicyano-1-(4-methoxyphenyl)ethylphosphonate (5j): Yellow solid, M. P. $155-157^{\circ} \mathrm{C}$, $[\alpha]_{\mathrm{D}}-26.8\left(\mathrm{c} 1.0, \mathrm{CHCl}_{3}\right)$; the title compound was prepared according to the general procedure, as described above in $(287 \mathrm{mg}) 82 \%$ yield. The structure of compound was confirmed by comparing spectral data, which were found to be identical with those described in literature 13a. HPLC: 76\% ee [Determined by chiral HPLC with Whelk-O1 $(25 \mathrm{~cm} \times 4.6 \mathrm{~mm}), 10 \%$ $\mathrm{EtOH} /$ hexane, flow rate $1.0 \mathrm{~mL} / \mathrm{min}, \lambda=245 \mathrm{~nm} ; \mathrm{t}_{\mathrm{R}}$ $($ major $\left.)=35.1 \mathrm{~min}, \mathrm{t}_{\mathrm{R}}(\operatorname{minor})=42.3 \mathrm{~min}\right]$.

\section{4k Diethyl 2,2-dicyano-1-cyclohexylethylphosphonate}

(5k): Colourless Solid, (Yield $298 \mathrm{mg} ; 73 \%$ ), M. P. $159-160^{\circ} \mathrm{C},[\alpha]_{\mathrm{D}}-38.2\left(\mathrm{c} 1.0, \mathrm{CHCl}_{3}\right) ;{ }^{1} \mathrm{H} \mathrm{NMR}$ (300 MHz, $\left.\mathrm{CDCl}_{3}\right): \delta$ 4.21-4.59 (m, 5H), 4.01 (dd, $\left.J^{\mathrm{H}-\mathrm{P}}=24.1 \mathrm{~Hz}, J^{\mathrm{H}-\mathrm{H}}=7.6 \mathrm{~Hz}, 1 \mathrm{H}\right), 2.04-2,13(\mathrm{~m}$, $1 \mathrm{H}), 1.55-1.82(\mathrm{~m}, 6 \mathrm{H}), 1.36-1.53(\mathrm{~m}, 4 \mathrm{H}), 1.27(\mathrm{t}$, $\left.J^{\mathrm{H}-\mathrm{H}}=6.3 \mathrm{~Hz}, 3 \mathrm{H}\right), 1.13\left(\mathrm{t}, J^{\mathrm{H}-\mathrm{H}}=6.3 \mathrm{~Hz}, 3 \mathrm{H}\right)$; ${ }^{13} \mathrm{C}-\mathrm{NMR}\left(75 \mathrm{MHz}, \mathrm{CDCl}_{3}\right): \delta 113.8\left(\mathrm{~d}, J^{\mathrm{C}-\mathrm{P}}=\right.$ $10.8 \mathrm{~Hz}), 62.8\left(\mathrm{~d}, J^{\mathrm{C}-\mathrm{P}}=4.9 \mathrm{~Hz}\right), 42.1\left(\mathrm{~d}, J^{\mathrm{C}-\mathrm{P}}=\right.$ $145.1 \mathrm{~Hz}), 30.4,29.8,28.2,27.2,16.1\left(\mathrm{~d}, J^{\mathrm{C}-\mathrm{P}}=\right.$ $5.3 \mathrm{~Hz}$ ); GC-MS: $m / z 298$ (M+); Elemental Analysis: $\mathrm{C}_{14} \mathrm{H}_{23} \mathrm{~N}_{2} \mathrm{O}_{3} \mathrm{P}: \mathrm{C}, 56.37$; H, 7.77; N, 9.39; Found C, 56.41; H, 7.75; N, 9.36. HPLC: $61 \%$ ee. [Determined by chiral HPLC with Whelk-O1 $(25 \mathrm{~cm} \times 4.6 \mathrm{~mm})$, $10 \% \mathrm{EtOH} / \mathrm{Hexane}$, Flow rate $1.0 \mathrm{~mL} / \mathrm{min}, \lambda=$ $245 \mathrm{~nm} ; \mathrm{t}_{\mathrm{R}}($ minor $)=25.2 \mathrm{~min}, \mathrm{t}_{\mathrm{R}}($ major $\left.)=32.4 \mathrm{~min}\right]$. 

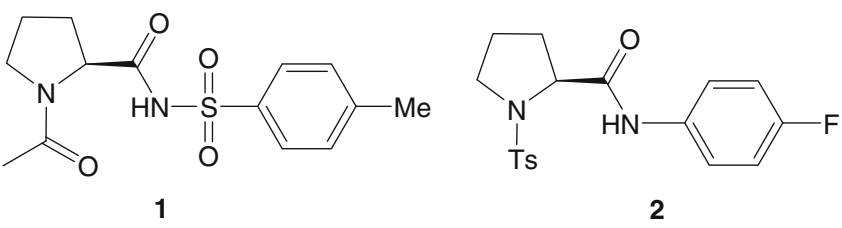

Figure 1. $L$-proline-based organocatalysts.

2.41 Diethyl 1,1-dicyano-3-methylpentan-2-ylphosphonate (5l): Colourless solid, (Yield $313 \mathrm{mg}$; 69\%), M. P. $121-123^{\circ} \mathrm{C},[\alpha]_{\mathrm{D}}-26.4\left(\mathrm{c} 1.0, \mathrm{CHCl}_{3}\right) ;{ }^{1} \mathrm{H}$ NMR $\left(300 \mathrm{MHz}, \mathrm{CDCl}_{3}\right): \delta$ 4.10-4.39 (m, 4H), 3.92 $\left(\mathrm{dd}, J^{\mathrm{H}-\mathrm{P}}=22.7 \mathrm{~Hz}, J^{\mathrm{H}-\mathrm{H}}=7.4 \mathrm{~Hz}, 2 \mathrm{H}\right), 1.99-$ $2.22(\mathrm{~m}, 3 \mathrm{H}), 1.60\left(\mathrm{~d}, J^{\mathrm{H}-\mathrm{H}}=7.6 \mathrm{~Hz}, 3 \mathrm{H}\right), 1.30(\mathrm{t}$, $\left.J^{\mathrm{H}-\mathrm{H}}=6.9 \mathrm{~Hz}, 3 \mathrm{H}\right), 1.18\left(\mathrm{t}, J^{\mathrm{H}-\mathrm{H}}=6.9 \mathrm{~Hz}, 3 \mathrm{H}\right), 0.87$ $\left(\mathrm{t}, J^{\mathrm{H}-\mathrm{H}}=7.3 \mathrm{~Hz}, 3 \mathrm{H}\right) ;{ }^{13} \mathrm{C}-\mathrm{NMR}\left(75 \mathrm{MHz}, \mathrm{CDCl}_{3}\right)$ : $\delta 113.8\left(\mathrm{~d}, J^{\mathrm{C}-\mathrm{P}}=11.9 \mathrm{~Hz}\right), 61.9\left(\mathrm{~d}, J^{\mathrm{C}-\mathrm{P}}=4.8 \mathrm{~Hz}\right)$, $42.2\left(\mathrm{~d}, J^{\mathrm{H}-\mathrm{H}}=143.9 \mathrm{~Hz}\right), 32.6,29.4,20.0,16.2$ $\left(\mathrm{d}, J^{\mathrm{C}-\mathrm{P}}=5.7 \mathrm{~Hz}\right), 10.1$; GC-MS: $m / z 272(\mathrm{M}+)$; Elemental Analysis: $\mathrm{C}_{12} \mathrm{H}_{21} \mathrm{~N}_{2} \mathrm{O}_{3} \mathrm{P}: \mathrm{C}, 52.93 ; \mathrm{H}, 7.77$; N, 10.29; Found C, 52.89; H, 7.79; N, 10.32. HPLC:
55\% ee. [Determined by chiral HPLC with WhelkO1 $(25 \mathrm{~cm} \times 4.6 \mathrm{~mm}), 10 \% \mathrm{EtOH} /$ hexane, flow rate $1.0 \mathrm{~mL} / \mathrm{min}, \lambda=245 \mathrm{~nm} ; \mathrm{t}_{\mathrm{R}}$ (minor) $=27.9 \mathrm{~min}, \mathrm{t}_{\mathrm{R}}$ (major) $=42.1 \mathrm{~min}]$.

\section{4m Diethyl 1,1-dicyanopentan-2-ylphosphonate} (5m): White solid, (Yield $275 \mathrm{mg} ; 64 \%$ ), M. P. $88-90{ }^{\circ} \mathrm{C},[\alpha]_{\mathrm{D}}-14.7$ (c $\left.1.0, \mathrm{CHCl}_{3}\right) ;{ }^{1} \mathrm{H}$ NMR $\left(300 \mathrm{MHz}, \mathrm{CDCl}_{3}\right): \delta 4.31-4.63(\mathrm{~m}, 4 \mathrm{H}), 4.15(\mathrm{dd}$, $\left.J^{\mathrm{H}-\mathrm{P}}=23.2 \mathrm{~Hz}, J^{\mathrm{H}-\mathrm{H}}=7.4 \mathrm{~Hz}, 1 \mathrm{H}\right), 1.71-1.88(\mathrm{~m}$, $1 \mathrm{H}), 1.25-1.49(\mathrm{~m}, 6 \mathrm{H}$ (aliphatic and ester)), 1.19 $\left(\mathrm{t}, J^{\mathrm{H}-\mathrm{H}}=7.3 \mathrm{~Hz}, 3 \mathrm{H}\right), 0.88\left(\mathrm{t}, J^{\mathrm{H}-\mathrm{H}}=8.7 \mathrm{~Hz}\right.$, 3H); ${ }^{13} \mathrm{C}-\mathrm{NMR} \quad\left(75 \mathrm{MHz}, \mathrm{CDCl}_{3}\right): \delta 113.6(\mathrm{~d}$, $\left.J^{\mathrm{C}-\mathrm{P}}=11.2 \mathrm{~Hz}\right), 62.7\left(\mathrm{~d}, J^{\mathrm{C}-\mathrm{P}}=5.1 \mathrm{~Hz}\right), 42.5(\mathrm{~d}$, $\left.J^{\mathrm{C}-\mathrm{P}}=144.3 \mathrm{~Hz}\right), 30.9,29.8,28.2,27.1,15.8(\mathrm{~d}$, $\left.J^{\mathrm{C}-\mathrm{P}}=6.2 \mathrm{~Hz}\right)$; GC-MS: $m / z 258(\mathrm{M}+)$; Elemental Analysis: $\mathrm{C}_{11} \mathrm{H}_{19} \mathrm{~N}_{2} \mathrm{O}_{3} \mathrm{P}: \mathrm{C}, 51.16 ; \mathrm{H}, 7.42 ; \mathrm{N}, 10.85 ;$; Found C, 51.19; H, 7.39; N, 10.83. HPLC: $53 \%$ ee. [Determined by chiral HPLC with Whelk-O1 $(25 \mathrm{~cm}$ $\times 4.6 \mathrm{~mm}), 10 \% \mathrm{EtOH} /$ hexane, flow rate $1.0 \mathrm{~mL} / \mathrm{min}$, $\lambda=245 \mathrm{~nm} ; \mathrm{t}_{\mathrm{R}}($ minor $)=20.6$ min, $\mathrm{t}_{\mathrm{R}}($ major $)=$ $23.1 \mathrm{~min}]$.

Table 1. Effect of solvents and catalytic loading on synthesis of diethyl 1-(4-chlorophenyl)-2,2-dicyanoethylphosphonate. ${ }^{\mathrm{d}}$
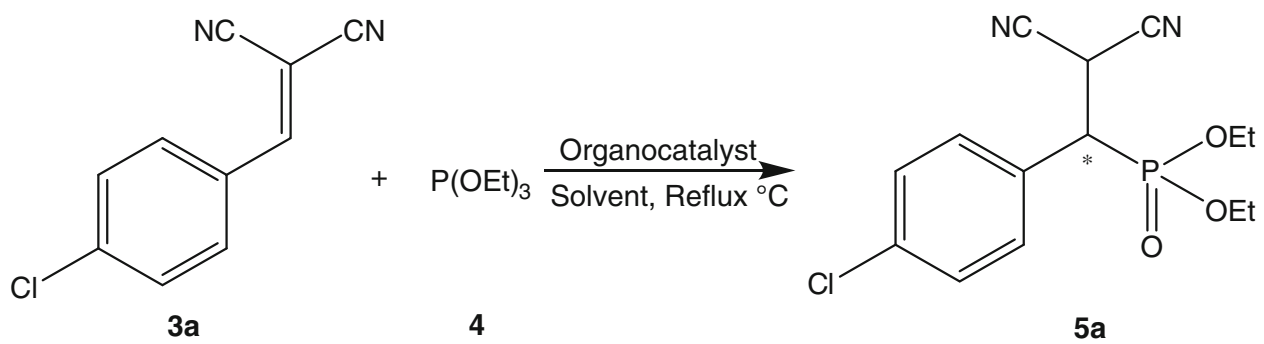

\begin{tabular}{ccccccc}
\hline Entry & Catalyst & Solvent & Mol $\%$ & Time (h) & Yield $^{\mathrm{a}}(\%)$ & $e e^{\mathrm{b}} \%$ \\
\hline 1 & 1 & Water & 10 & 36 & 39 & 54 \\
2 & 2 & Water & 10 & 36 & 35 & 52 \\
3 & 1 & Ethanol & 10 & 12 & 74 & 68 \\
4 & 2 & Ethanol & 10 & 17 & 57 & 61 \\
5 & 1 & Tolune & 10 & 24 & 53 & 29 \\
6 & 1 & Chloroform & 10 & 26 & 49 & 34 \\
7 & 1 & Dichloromethane & 10 & 28 & 40 & 66 \\
8 & 1 & Ethanol & 7.5 & 19 & 67 & 78 \\
9 & 1 & Ethanol & 12 & 7 & 85 & $\mathrm{Nd}$ \\
10 & 1 & Ethanol & 15 & 18 & 71 & $\mathrm{Nd}$ \\
$11^{\mathrm{c}}$ & 1 & Ethanol & 12 & 36 & 34 & 23 \\
12 & No catalyst & Ethanol & - & 36 & & \\
\hline
\end{tabular}

asolated yield

${ }^{\mathrm{b}}$ Determined by chiral HPLC analysis

${ }^{\mathrm{c}}$ The reaction was performed at room temperature conditions

${ }^{\mathrm{d}}$ The reactions were performed at reflux temperature conditions. The Progress of reaction was monitored by thin layer chromatography

Nd, Not determined 


\section{Result and discussion}

As part of our ongoing program directed toward the development organocatalytic pathways, we studied the synthesis of $\beta$-phosphonomalonates via phosphaMichael addition of trialkyl phosphites to alkylidene malonates. The reports documented in literature shows that the acidic conditions are not appropriate for the phospha-Michael addition, the reaction results in low yield of the product after a long reaction time. ${ }^{15-18}$ This observation prompted us to examine the feasibility of the synthesis of $\beta$-phosphonomalonates by phospha-Michael addition of triethyl phosphite to $\alpha, \beta$-unsaturated malonates in the presence of organocatalysts. On the basis of the success of our previous results ${ }^{19}$ on hydrogen bonding organocatalysis by neutral chiral pyrrolidine compounds (figure 1) and escalating our research in organocatalysis, we sought to study the asymmetric synthesis of $\beta$-phosphonomalonates by these pyrrolidine-based organocatalysts ( $\mathbf{1}$ and $\mathbf{2})$, exclusively via hydrogen-bonding interaction.

We started with phospha-Michael addition of triethyl phosphate 4 to 2-(4-chlorobenzylidene) malononitrile 3a as a model reaction in the presence of $10 \mathrm{~mol} \%$ of organocatalyst $\mathbf{1}$ and $\mathbf{2}$ in a variety of solvents.

When reaction was carried out in water by using catalyst $\mathbf{1}$ or $\mathbf{2}$ the yield obtained for products were not so good with poor enantioselectivity and reaction time was also much longer (table 1, entries 1 and 2). Improved results were obtained when solvent ethanol was used (table 1, entries 3 and 4). Organocatalyst (S)-1acetylpyrrolidine-2-carboxamide (1) was found to be

Table 2. Synthesis of derivatives of $\beta$-malonophosphonates using various aromatic aldehydes. ${ }^{\mathrm{c}}$<smiles>[R]C=C(C#N)C#N</smiles>

$3(\mathrm{a}-\mathrm{j})$<smiles>[R]C(C(C#N)C#N)P(=O)(OCC)OCC</smiles>

$5(a-j)$

\begin{tabular}{|c|c|c|c|c|c|}
\hline Entry & $\mathrm{R}$ & Product & Time $(\mathrm{h})$ & Yield $^{\mathrm{a}}(\%)$ & $e e^{\mathrm{b}}(\%)$ \\
\hline 1 & $4-\mathrm{Cl}-\mathrm{C}_{6} \mathrm{H}_{4}$ & $5 a$ & 7.00 & 85 & 78 \\
\hline 2 & 4- $\mathrm{NO}_{2}-\mathrm{C}_{6} \mathrm{H}_{4}$ & $5 b$ & 9.00 & 83 & 71 \\
\hline 3 & $3-\mathrm{NO}_{2}-\mathrm{C}_{6} \mathrm{H}_{4}$ & $5 c$ & 8.00 & 85 & 67 \\
\hline 4 & $4-\mathrm{F}-\mathrm{C}_{6} \mathrm{H}_{4}$ & $5 \mathrm{~d}$ & 9.00 & 81 & 69 \\
\hline 5 & $3-\mathrm{Cl}-\mathrm{C}_{6} \mathrm{H}_{4}$ & $5 e$ & 7.00 & 78 & 76 \\
\hline 6 & $4-\mathrm{OH}-\mathrm{C}_{6} \mathrm{H}_{4}$ & $5 \mathrm{f}$ & 7.00 & 85 & 59 \\
\hline 7 & $2-\mathrm{OH}-\mathrm{C}_{6} \mathrm{H}_{4}$ & $5 \mathrm{~g}$ & 7.30 & 80 & 63 \\
\hline 8 & $-\mathrm{C}_{6} \mathrm{H}_{5}$ & $5 \mathrm{~h}$ & 7.00 & 77 & 59 \\
\hline 9 & $4-\mathrm{Me}-\mathrm{C}_{6} \mathrm{H}_{4}$ & $5 \mathrm{i}$ & 6.30 & 79 & 65 \\
\hline 10 & $4-\mathrm{OCH}_{3}-\mathrm{C}_{6} \mathrm{H}_{4}$ & $5 \mathrm{j}$ & 6.30 & 82 & 76 \\
\hline 11 & & $5 \mathrm{k}$ & 10.00 & 73 & 61 \\
\hline 12 & & 51 & 9.30 & 69 & 55 \\
\hline 13 & & $5 \mathrm{~m}$ & 10.00 & 64 & 53 \\
\hline
\end{tabular}

asolated yield

${ }^{b}$ Determined by chiral HPLC analysis

${ }^{\mathrm{c}}$ The Progress of reaction was monitored by thin layer chromatography 
superior over the catalyst (S)- $N$-(4-fluorophenyl)-1tosylpyrrolidine-2-carboxamide (2) and solvent ethanol was the best choice for synthesis of $\beta$-phosphonomalonates as best results were obtained in ethanol as solvent, the other solvent furnished poor results (table 1, entries 5-7). So, further optimization of reaction was carried out using organocatalyst $\mathbf{1}$ in the solvent ethanol.

Next, the reaction was studied at various catalytic loading to find suitable quantity for the reaction. The results of these studies are summarized in table 1. As shown in table 1 , the reaction proceeded well in the presence of $12 \mathrm{~mol} \%$ of the organocatalyst 1 in ethanol as solvent at reflux conditions and produced corresponding $\beta$-phosphonomalonate (5a) in high yield and enantioselectivity (table 1 , entries $8-10$ ). When the reaction was carried out at room temperature, the yield of the product decreased significantly and enantioselectivity was not determined (table 1, entry 11). In order to show the role of the catalyst, similar reactions in the absence of the catalyst was also examined. Under these conditions, the reaction led to the formation of the desired product in low yields after a long reaction time (table 1, entry 12).

In order to find the scope and limitation of this method, the reaction of triethyl phosphite with various types of $\alpha, \beta$-unsaturated malonates in the presence of organocatalyst 1 using optimized reaction conditions was investigated. The results are summarized in table 2 .

From table 2, it can be concluded that the catalytic phospha-Michael addition of triethyl phosphite with benzylidenemalonitriles containing different electrondonating and electron-withdrawing groups proceeded well and the desired products $(\mathbf{5} \mathbf{a}-\mathbf{j})$ were obtained in good to high yields. In the case of aliphatic compounds reaction proceeded slowly to offer moderate yield and enantioselectivity for products $(\mathbf{5 k}-\mathbf{m})$.

The mechanism of reaction is not sure but it is assumed that, organocatalyst activate the alkylidinemalanonitrile and increase the electrophilicity of carbon atom followed by the addition of phosphite nucleophile which leads to desired product. Hydrogen bonding and non-bonding interaction (in case of electro donating substituents) are most likely to be responsible for activation of substrate. The driving force for chirality could be steric hindrance, hydrogen bonding and non-bonding interaction.

\section{Conclusion}

In summary, the synthesized organocatalysts (1 and $\mathbf{2})$ were successfully used to catalyse asymmetric synthesis of $\beta$-malonophosphonates; especially the organocatalyst
(S)-N-(4-fluorophenyl)-1-tosylpyrrolidine-2-carboxamide (1) was more effective to give a series of optically active $\beta$-malonophosphonates in good enantiomeric purity and high yield. The methodology described here represents one of the most straightforward routes for synthesis of optically active $\beta$-malonophosphonates.

\section{Supporting information}

Includes ${ }^{1} \mathrm{H}$ NMR, ${ }^{13} \mathrm{C}$ NMR, ${ }^{31} \mathrm{P}$ NMR and GC-MS spectra for representatives, and HPLC chromatograms for all compounds can be seen at www.ias.ac.in/ chemsci website.

\section{Acknowledgements}

We thank Dr. P L More, Dnyanopasak College, Parbhani for providing necessary facilities, and also Indian Institute of Chemical Technology (IICT), Hyderabad for providing spectral data.

\section{References}

1. Enders D, Saint-Dizier A, Lannou M I and Lenzen A 2006 Eur. J. Org. Chem. 29

2. Zhang S, Gangal G and Uludag H 2007 Chem. Soc. Rev. 36507

3. Zhou Y G 2007 Acc. Chem. Res. 401357

4. (a) Seto H and Kuzuyama T 1999 Nat. Prod. Rep. 16 589; (b) Fields S C 1999 Tetrahedron 5512237

5. Pudovik A N and Konovalova I V 1979 Synthesis 81

6. Arbuzov A B 1964 Pure Appl. Chem. 9307

7. Horiguchi M and Kandastu M 1959 Nature 184901

8. Bodalski R and Pietrusiewicz K 1972 Tetrahedron Lett. 134209

9. Simoni D, Invidiata F P, Manferdini M, Lampronti I, Rondanin R, Roberti M and Pollini G P 1998 Tetrahedron Lett. 397615

10. Hindersinn R R and Ludington R S 1965 J. Org. Chem. 304020

11. Stockland R A, Taylor R I, Thompson L E and Patel P B 2005 Org. Lett. 7851

12. Han L B and Zhao C Q 2005 J. Org. Chem. 7010121

13. (a) Hosseini-Sarvari M and Etemad S 2008 Tetrahedron 64 5519; (b) Hosseini-Sarvari M and Tavakolian M 2012 New J. Chem. 361014

14. Sobhani S, Parizi Z P and Rezazadeh S $2011 \mathrm{~J}$. Organomet. Chem. $\mathbf{6 9 6} 813$

15. Shulyupin M O, Kazankova M A and Beletskaya I P 2002 Org. Lett. 4761

16. Xu Q and Han L B 2008 Org. Lett. 82099

17. Janecki T, Kedzia J and Wasek T 2009 Synthesis 1227

18. Kolla S R and Lee Y R 2012 Tetrahedron 68226

19. (a) Thorat P B, Goswami S V, Khade B C and Bhusare S R 2012 Tetrahedron Asymmetry 23 1320; (b) Thorat P B, Goswami S V, Khade B C and Bhusare S R 2012 Tetrahedron Lett. 536083 